\title{
Maintenance chemotherapy is effective in patients with metastatic triple negative breast cancer after first-line platinum-based chemotherapy
}

\author{
Yang Chen, Jian Zhang, Xi-Chun Hu, Bi-Yun Wang, Zhong-Hua Wang, Lei-Ping Wang, Jun Cao, \\ Zhong-Hua Tao, Yi-Qun Du, Yan-Nan Zhao, Cheng-Cheng Gong, Jia Jin
}

Department of Medical Oncology, Fudan University Shanghai Cancer Center, Department of Oncology, Shanghai Medical College, Fudan University, Shanghai, China

Contributions: (I) Conception and design: XC Hu, J Zhang, Y Chen; (II) Administrative support: XC Hu, J Zhang; (III) Provision of study materials or patients: All authors; (IV) Collection and assembly of data: Y Chen;(V) Data analysis and interpretation: Y Chen; (VI) Manuscript writing: All authors; (VII) Final approval of manuscript: All authors.

Correspondence to: Prof. Xi-Chun Hu; Jian Zhang, MD. Department of Medical Oncology, Fudan University Shanghai Cancer Center, Department of Oncology, Shanghai Medical College, Fudan University, Dong'an Road 270, Shanghai, China. Email: xchu2009@hotmail.com; syner2000@163.com.

Background: Platinum-based chemotherapy (PBCT) has gained an important position as a first-line treatment for metastatic triple-negative breast cancer (mTNBC). We assessed whether maintenance chemotherapy maintenance was superior to observation after first-line PBCT in patients with mTNBC.

Methods: A total of 265 patients with mTNBC who had exhibited non-PD after 4-6 cycles of firstline PBCT at the Fudan University Shanghai Cancer Center from January 2008 to April 2019 were retrospectively analyzed. 107 patients who did not receive additional treatment were defined as the control observation group, and the remaining 158 patients who continued to receive maintenance therapy were defined as the maintenance treatment group.

Results: The median progression-free survival (PFS) time in the maintenance group was 9.63 months, which was significantly longer than the PFS time of 7.47 months in the observation group (HR 0.49, $95 \%$ CI: $0.37-0.67, \mathrm{P}<0.0001)$. The median overall survival (OS) of the observation group and the maintenance group was 25.37 months and 31.27 months, respectively (HR 0.65, 95\% CI: 0.44-0.95, P=0.019). The survival benefit was still present after adjusting baseline characteristics. Moreover, multivariate analyses suggested that maintenance chemotherapy is an independent predictive factor for both PFS and OS. Interaction and stratified analyses showed no difference in the PFS with between the single-drug maintenance strategy, single agent or doublet group and the doublet-drug maintenance group. The most common adverse event in this study was hematologic toxicity. Except for hand-foot syndrome ( 0 vs. 7.6\%, P=0.004), the incidence of other adverse events was not significantly different between the observation and maintenance groups.

Conclusions: After achieving non-PD with the first-line PBCT in mTNBC patients, chemotherapy maintenance may provide OS benefit prior to the era of biologicals.

Keywords: Metastatic breast cancer (MBC); triple-negative breast cancer (TNBC); maintenance therapy; platinums

Submitted Mar 07, 2020. Accepted for publication Jun 24, 2020.

doi: 10.21037/apm-20-578

View this article at: http://dx.doi.org/10.21037/apm-20-578 


\section{Introduction}

Triple-negative breast cancer (TNBC), which lacks expression of the estrogen receptor (ER), progesterone receptor (PR) and human epidermal growth factor receptor-2 (HER-2), accounts for $\sim 12-17 \%$ of all breast cancers (1). TNBC is associated with a high rate of recurrence, distant metastasis and visceral metastasis (2-4). The median survival for metastatic TNBC (mTNBC) is only approximately 1 year $(5,6)$. Due to insufficient therapeutic targets, the treatment of TNBC is a major clinical challenge. The main treatment option for mTNBC is chemotherapy, and platinum-based chemotherapy (PBCT) plays an important role in first-line chemotherapy for $\mathrm{mTNBC}$.

Over the past decade, the development of PBCT has led to a gradual increase in the survival of patients with mTNBC. In 2007, when platinums were not used as the first-line chemotherapy for mTNBC, the median survival of mTNBC was only 9 months (3). After 7 years, a phase III clinical study by O'Shaughnessy et al. showed that gemcitabine plus carboplatin (GC) extended the median progression-free survival (PFS) and overall survival (OS) of mTNBC patients to 4.1 and 11.1 months, respectively (7). In another randomized phase III study, the CBCSG006 trial, which indicated that cisplatin plus gemcitabine (GP) could be an alternative, or even the preferred, first-line chemotherapy strategy for patients with mTNBC, the median PFS in the GP group reached 7.73 months [95\% confidence interval (CI): 6.16-9.30] (8). With these two studies, the important role of platinums in the treatment of mTNBC has been gradually accepted.

Maintenance chemotherapy is chemotherapy administered to patients who have achieved complete remission (CR), partial remission (PR), or disease stabilization (SD) after an initial treatment with chemotherapy with a standard duration (6-8 courses) (9). Some clinical studies have found that patients with HER2positive and hormone-positive disease can achieve a clinical benefit from maintenance therapy $(10,11)$. However, currently, there has been no prospective clinical study that focused on maintenance chemotherapy after first-line PBCT in mTNBC. In 2013, the phase III clinical study KCSGBR-0702 evaluated whether maintenance chemotherapy with paclitaxel plus gemcitabine (PG) was superior to observation in patients with metastatic breast cancer (MBC) who achieved disease control with an initial six cycles of PG as their first-line treatment. This study observed a longer
PFS (7.5 vs. 3.8 months, respectively; $\mathrm{P}=0.026)$ and $\mathrm{OS}(32.3$ vs. 23.5 months, respectively; $\mathrm{P}=0.047$ ) in the maintenance group than in the observation group (12). The subgroup analysis in this study showed that the PFS benefits of maintenance chemotherapy were observed in patients with hormone receptor-negative disease (95\% CI: 0.30 to 0.90; $\mathrm{P}=0.019$ ). Moreover, some retrospective studies affirmed the positive role of maintenance chemotherapy in mTNBC $(13,14)$. To determine whether maintenance chemotherapy is a reasonable strategy to prolong PFS and OS in patients with $\mathrm{mTNBC}$ who achieved disease control with firstline $\mathrm{PBCT}$, we retrospectively reviewed a large cohort of mTNBC patients at the Fudan University Shanghai Cancer Center (FUSCC) to evaluate the efficacy and safety of the maintenance chemotherapy. We present the following article in accordance with the STROBE reporting checklist (available at http://dx.doi.org/10.21037/apm-20-578).

\section{Methods}

\section{Eligibility criteria}

The major inclusion criteria were as follows: female sex; age $\geq 18$ years; TNBC that was histologically confirmed using the primary tumor (ER-negative and PR-negative statuses were defined as $<1 \%$ according to the new College of American Pathologists guidelines at that time; HER2-negative status was defined as a score of 0 or 1 by immunohistochemistry or as the absence of HER2 amplification (ratio <2.2) by fluorescence in situ hybridization analysis); at least one measurable lesion by MRI or CT according to the Response Evaluation Criteria in Solid Tumors (RECIST) guideline version 1.1; histopathology that confirmed the presence of unresectable recurrent metastases, including newly diagnosed stage IV breast cancer; an Eastern Cooperative Oncology Group score $\leq 1$; completion of $4-6$ cycles of first-line PBCT; and disease control (CR, PR, or SD).

Patients were excluded if the primary tumor receptor status data were incomplete or if a subsequent relapse was known to be positive for ER, PR or HER2. Other exclusion criteria included pregnant or lactating women; evidence of CNS metastasis; history of another malignancy within the last five years, except cured basal cell carcinoma of the skin, carcinoma in situ of the uterine cervix or contralateral breast cancer; serious, uncontrolled, intercurrent infection; serious cardiovascular, liver and kidney disease, severe bone marrow dysfunction or endocrine dysfunction. We also excluded 
cases with missing data. The number of cases in FUSCC during the study period determined the sample size.

\section{Therapy methods}

Patients received a 21-day cycle first-line doublet-drug regimen, which included GP, GC, and albumin-paclitaxel plus cisplatin (AP). Cisplatin was administered at a dose of $75 \mathrm{mg} / \mathrm{m}^{2}$ on day 1 in GP/AP. Carboplatin was administered at a dose of AUC 2 on day 1 in GC. Gemcitabine was administered at a dose of $1,250 \mathrm{mg} / \mathrm{m}^{2}$ in GP and $1,000 \mathrm{mg} / \mathrm{m}^{2}$ in GC on days 1 and 8 . Albumin-paclitaxel was administered at a dose of $125 \mathrm{mg} / \mathrm{m}^{2}$ on days 1 and 8 . The original doublet-drug maintenance chemotherapy regimen included GP and AP. The single-drug maintenance chemotherapy regimen included capecitabine and gemcitabine. Capecitabine was administered at a dose of $1.5 \mathrm{~g}$ twice daily on days $1-14$, followed by a $7-\mathrm{d}$ rest period. Gemcitabine was administered at a dose of $1,250 \mathrm{mg} / \mathrm{m}^{2}$ on days 1 and 8 .

\section{Efficacy and safety assessments}

The primary endpoint of this study was PFS, defined as the time from the start of the first-line treatment until disease progression or death. The secondary endpoints included OS and safety. OS was defined as the time from the start of the first-line treatment to death by any cause or was censored at the last date the patient was known to be alive. Adverse events were recorded before every cycle and graded according to the Common Terminology Criteria for Adverse Events (Version 4.0). The target lesion was assessed every 2 cycles using the RECIST guidelines (RECIST 1.1).

\section{Statistical methods}

PFS and OS were estimated using the Kaplan-Meier method, and comparisons between the two groups were performed using log-rank tests. Analyses of the treatment effects were adjusted with a multifactor Cox proportional hazard regression model. Baseline characteristics and incidence of adverse events were compared using Pearson's chi-square test and a $t$-test. All $\mathrm{P}$ values were calculated using a two-sided test and were considered statistically significant when $\mathrm{P}<0.05$. Interaction and stratified analyses were performed with EmpowerStats (http://www. empowerstats.com, X\&Y Solutions, Inc., Boston, MA,
USA). All statistical analyses and data calculations were conducted using SPSS software (version 22.0).

The study was conducted in accordance with the Declaration of Helsinki (as revised in 2013). This retrospective study was approved by Fudan University Shanghai Cancer Center Medical Ethics Committee (No. 1412142-14) and informed consent was taken from all the patients.

\section{Results}

\section{Patient characteristics}

A total of 265 patients treated at the FUSCC from January 2008 to April 2019 were enrolled in this study. After achieving disease control with 4-6 cycles of firstline PBCT, 107 patients who continued without additional treatment were defined as the control observation group for this study. In observation group, 84 (78.5\%) patients rested chemotherapy due to physicians' preference, 12 (11.2\%) patients due to patient's preference, and $11(10.3 \%)$ patients due to intolerant toxicity. The remaining 158 patients continued to receive maintenance therapy after completing 6 courses of first-line PBCT and were defined as the maintenance treatment group for this study. The doublet-drug regimens included in observation group were GP ( $n=81,75.7 \%)$, GC ( $n=8,7.5 \%)$, and AP ( $n=18,16.8 \%)$. $18(11.4 \%)$ and $33(20.9 \%)$ patients received capecitabine and gemcitabine maintenance therapy, respectively. The baseline characteristics of the patients were similar between the two groups (Table 1). Among all the patients, the oldest was 78 years old, the youngest was 29 years old, and the average age was 49.26 years. The numbers of patients aged $>40$ years in the observation and maintenance groups were $83(77.6 \%)$ and 125 (79.1\%), respectively, and approximately half of patients were premenopausal women. The numbers of patients who had achieved CR or PR after first-line chemotherapy were $66(61.7 \%)$ and $109(69.0 \%)$ in the observation and maintenance groups, respectively. The most common site of metastasis was the lymph node, and pleural metastasis was the least common event. More than half of patients had visceral metastasis.

\section{Efficacy analysis}

By April 15, 2019, a total of 217 (81.9\%) patients were documented to have disease progression or death. Among them, 91 patients $(85.0 \%)$ were in the observation group, 
Table 1 Patient characteristics in maintenance group and observation group

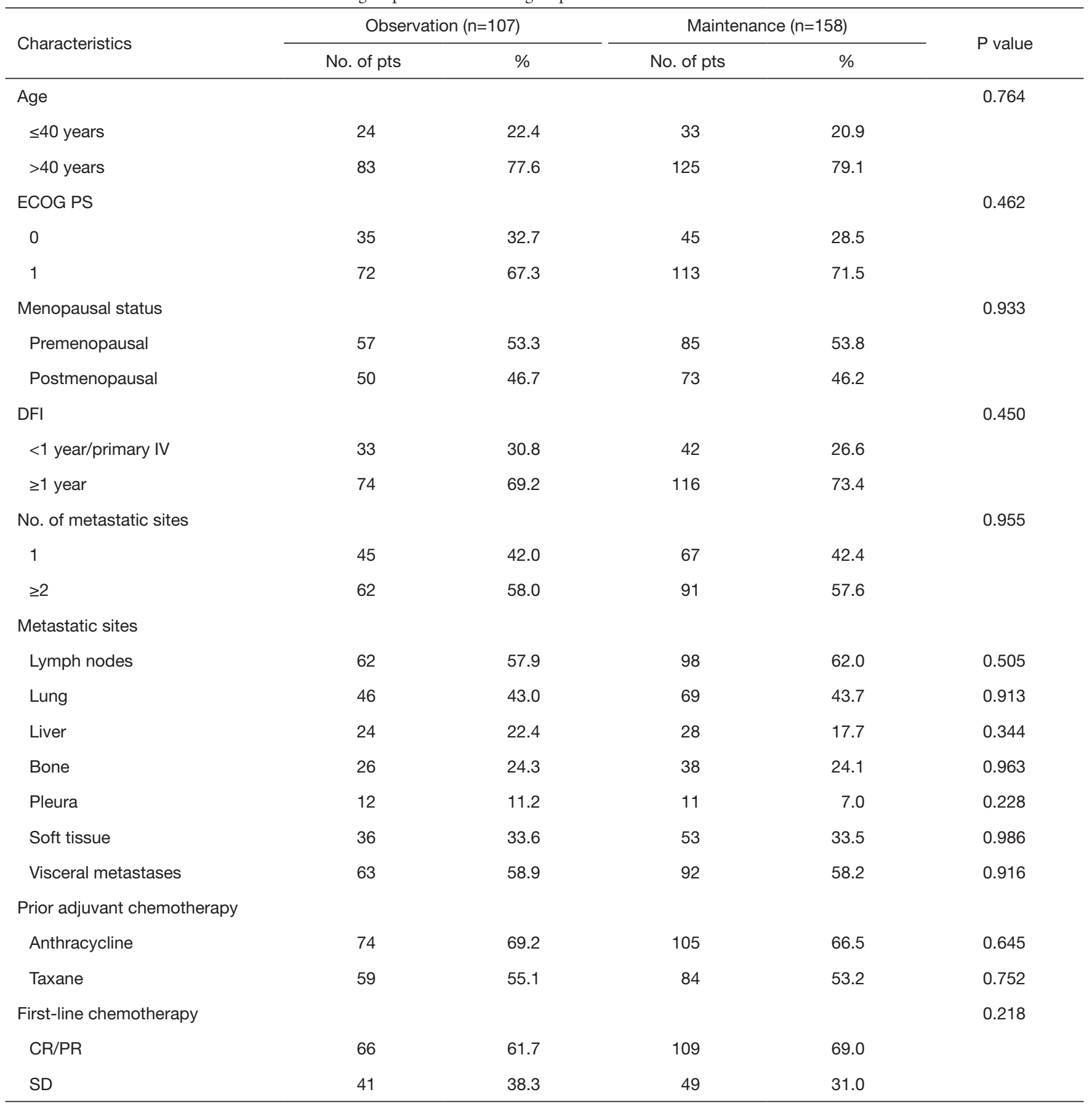

ECOG, Eastern Cooperative Oncology Group; PS, performance status; DFI, disease free interval; CR, complete remission; PR, partial remission; SD, disease stabilization.

and 126 patients $(79.7 \%)$ were in the maintenance group. The median PFS time in the maintenance group was 9.63 months, which was significantly longer than the median PFS time of 7.47 months in the observation group.
There was a significant difference between the PFS of the two groups [hazard ratio (HR) $0.49,95 \%$ CI: $0.37-0.67$, $\mathrm{P}<0.0001$; Figure 1A]. The median OS time was also shorter in the observation group than in the maintenance group 

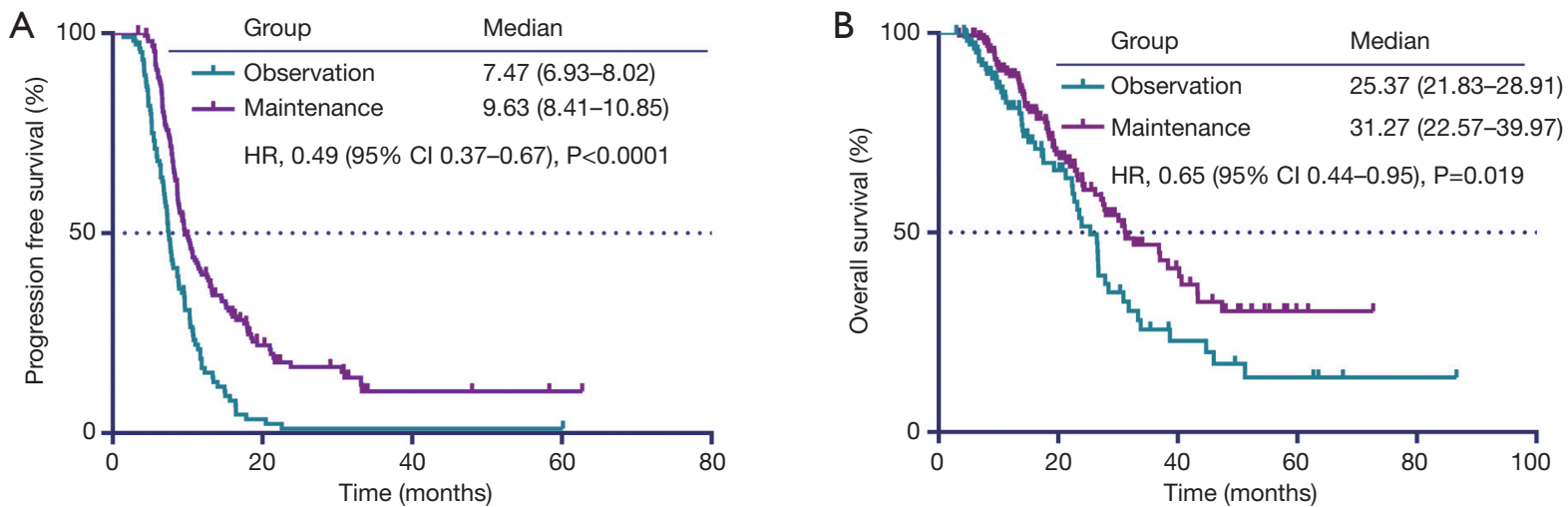

Figure 1 (A) Progression-free survival (PFS) and (B) overall survival in the maintenance and observation groups.

Table 2 Univariate and multivariate cox regression analysis of median PFS

\begin{tabular}{|c|c|c|c|c|c|c|}
\hline Characteristics & \multicolumn{3}{|c|}{ Univariate cox regression } & \multicolumn{3}{|c|}{ Multivariate cox regression } \\
\hline Age ( $\leq 40$ years, $>40$ years) & 0.97 & $0.70-1.34$ & 0.841 & & & \\
\hline ECOG PS $(0,1)$ & 1.22 & $0.86-1.73$ & 0.260 & & & \\
\hline Menopausal status (postmenopausal, premenopausal) & 1.26 & $0.97-1.65$ & 0.090 & & & \\
\hline Metastatic sites $(1, \geq 2)$ & 1.48 & $1.26-1.75$ & $<0.001$ & 1.42 & $1.12-1.69$ & $<0.001$ \\
\hline Visceral metastases & 1.33 & $1.01-1.75$ & 0.042 & 1.10 & $0.83-1.46$ & 0.502 \\
\hline First-line chemotherapy (CR/PR, SD) & 1.85 & $1.40-2.44$ & $<0.001$ & 1.86 & $1.40-2.46$ & $<0.001$ \\
\hline Observation/maintenance & 0.49 & $0.37-0.64$ & $<0.001$ & 0.48 & $0.36-0.63$ & $<0.001$ \\
\hline
\end{tabular}

ECOG, Eastern Cooperative Oncology Group; PS, performance status; HR, hazard ratio; Cl, confidence interval; CR, complete remission; $\mathrm{PR}$, partial remission; SD, disease stabilization.

(25.37 vs. 31.27 months, respectively; HR 0.65, 95\% CI: $0.44-0.95, \mathrm{P}=0.019$; Figure $1 B$ ).

To determine whether maintenance treatment was an independent factor for predicting PFS and OS, we evaluated several clinical features of patients using univariate analysis. The factors that significantly influenced PFS and OS in the univariate analysis included menstrual status, number of metastatic lesions, efficacy of first-line chemotherapy, visceral metastases, and maintenance treatment. Breast cancer in young women ( $\leq 40$ years) has greater morbidity and fatality than in older women, so we divided the patients into two groups at the age of 40 (15). We calculated the HRs of these factors using a multivariate Cox proportionalhazards regression model. The results showed that maintenance chemotherapy was an independent predictive factor for PFS (HR, 0.48; 95\% CI: 0.36-0.63; $\mathrm{P}<0.001$;
Table 2) and OS (HR, 0.68; 95\% CI: 0.47-0.99; $\mathrm{P}=0.044$; Table 3).

Exploratory analyses of PFS across the main clinical subgroups showed that all the subgroups favored the maintenance group (Figure 2). The single-drug $(\mathrm{n}=47)$ and doublet-drug maintenance regimens $(\mathrm{n}=111)$ showed no difference in effect on PFS, and this was consistent across subgroups (Figure 3).

\section{Toxicity analysis}

Table 4 presents the drug-related toxicities (according to the National Cancer Institute Common Terminology Criteria for Adverse Events) per patient that were observed. The calculation of adverse events started with first-line chemotherapy. The most common adverse event in the 
Table 3 Univariate and multivariate cox regression analysis of median OS

\begin{tabular}{|c|c|c|c|c|c|c|}
\hline Characteristics & \multicolumn{3}{|c|}{ Univariate Cox regression } & \multicolumn{3}{|c|}{ Multivariate Cox regression } \\
\hline Age ( $\leq 40$ years, $>40$ years) & 0.87 & $0.57-1.32$ & 0.500 & & & \\
\hline ECOG PS $(0,1)$ & 1.11 & $0.67-1.83$ & 0.684 & & & \\
\hline Menopausal status (premenopausal, postmenopausal) & 1.55 & $1.07-2.25$ & 0.021 & 1.48 & $1.01-2.15$ & 0.042 \\
\hline Metastatic sites $(1, \geq 2)$ & 1.60 & $1.10-2.33$ & 0.014 & 1.47 & $1.00-2.14$ & 0.048 \\
\hline Visceral metastases & 0.96 & $0.68-1.42$ & 0.918 & & & \\
\hline First-line chemotherapy (CR/PR, SD) & 1.59 & $1.09-2.32$ & 0.015 & 1.47 & $1.01-2.15$ & 0.045 \\
\hline Observation, maintenance & 0.65 & $0.45-0.94$ & 0.020 & 0.68 & $0.47-0.99$ & 0.044 \\
\hline
\end{tabular}

ECOG, Eastern Cooperative Oncology Group; PS, performance status; HR, hazard ratio; Cl, confidence interval; CR, complete remission; $\mathrm{PR}$, partial remission; SD, disease stabilization.

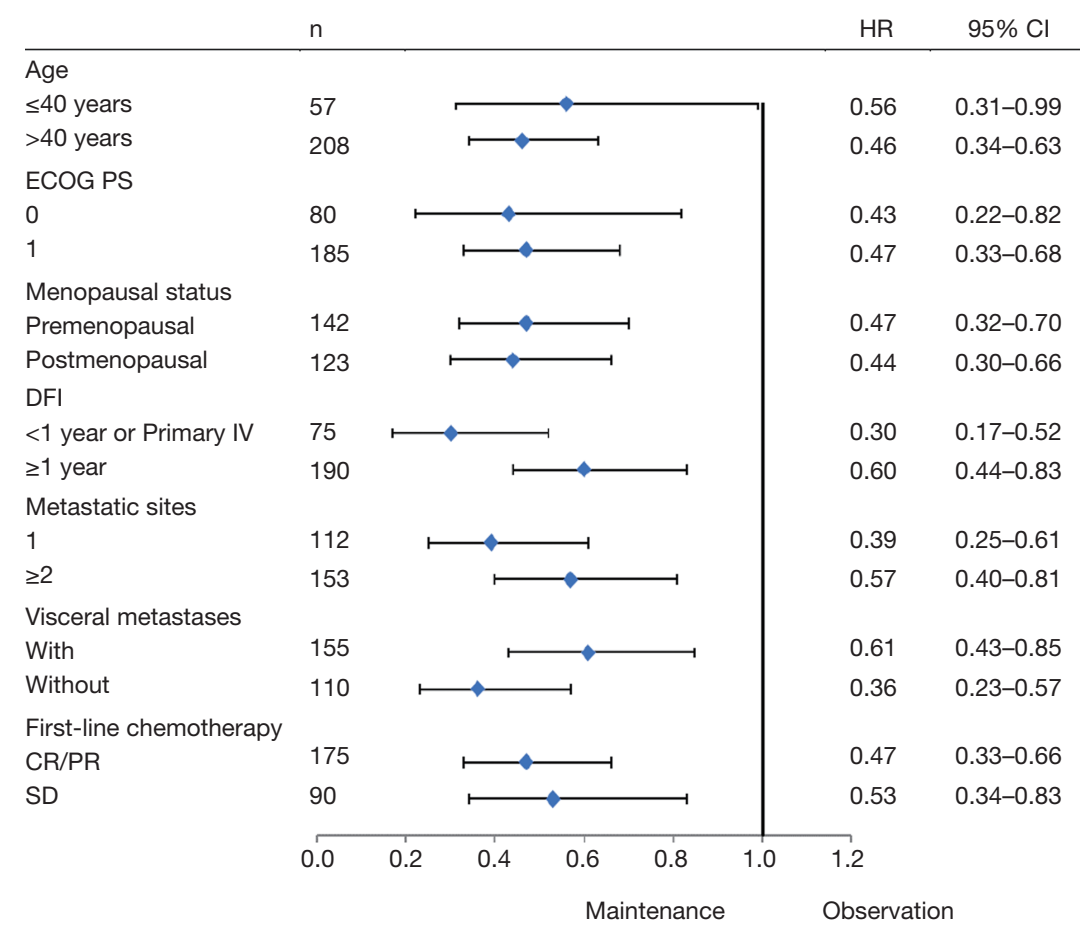

Figure 2 Forest plots (PFS analysis between observation and maintenance). PFS, progression-free survival; HR, hazard ratio; CI, confidence interval; ECOG, Eastern Cooperative Oncology Group; PS, performance status; DFI, disease free interval.

observation and maintenance groups was neutropenia $(82.2 \%$ vs. $83.5 \%)$. Other adverse events with high incidence were thrombocytopenia $(62.6 \%$ vs. $58.2 \%)$, anemia $(71.0 \%$ vs. $75.9 \%)$, nausea $(72.9 \%$ vs. $79.1 \%)$, and vomiting (61.7\% vs. 67.7\%). Except for hand-foot syndrome (0 vs. $7.6 \%, \mathrm{P}=0.004)$, there was no statistically significant difference in the incidence of other adverse events between the two groups.

\section{Discussion}

Although PBCT has shown advantages as the first-line 


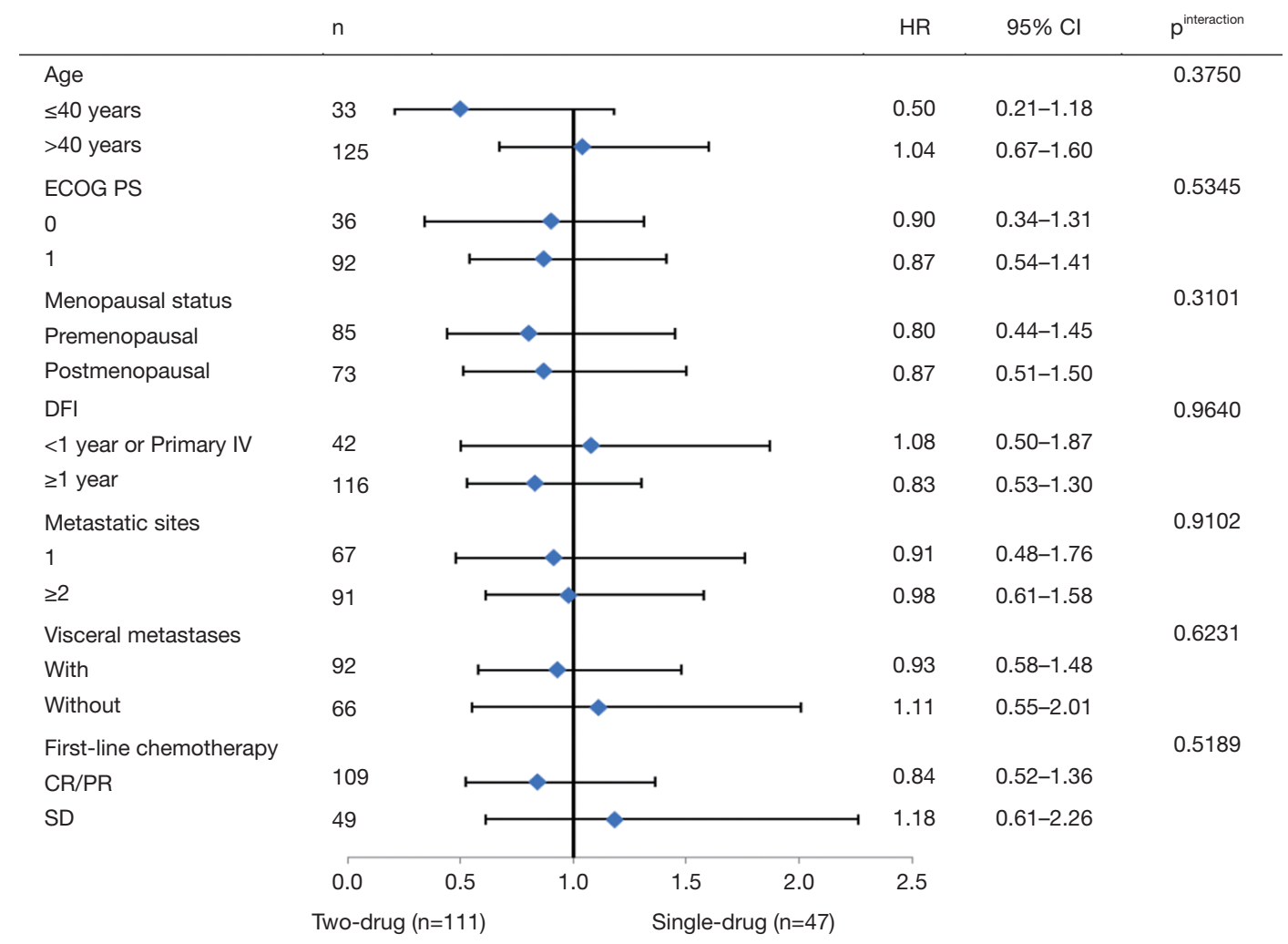

Figure 3 Forest plots (PFS analysis between two and single drug maintenance). PFS, progression-free survival; HR, hazard ratio; CI, confidence interval; ECOG, Eastern Cooperative Oncology Group; PS, performance status; DFI, disease free interval.

Table 4 Toxicity

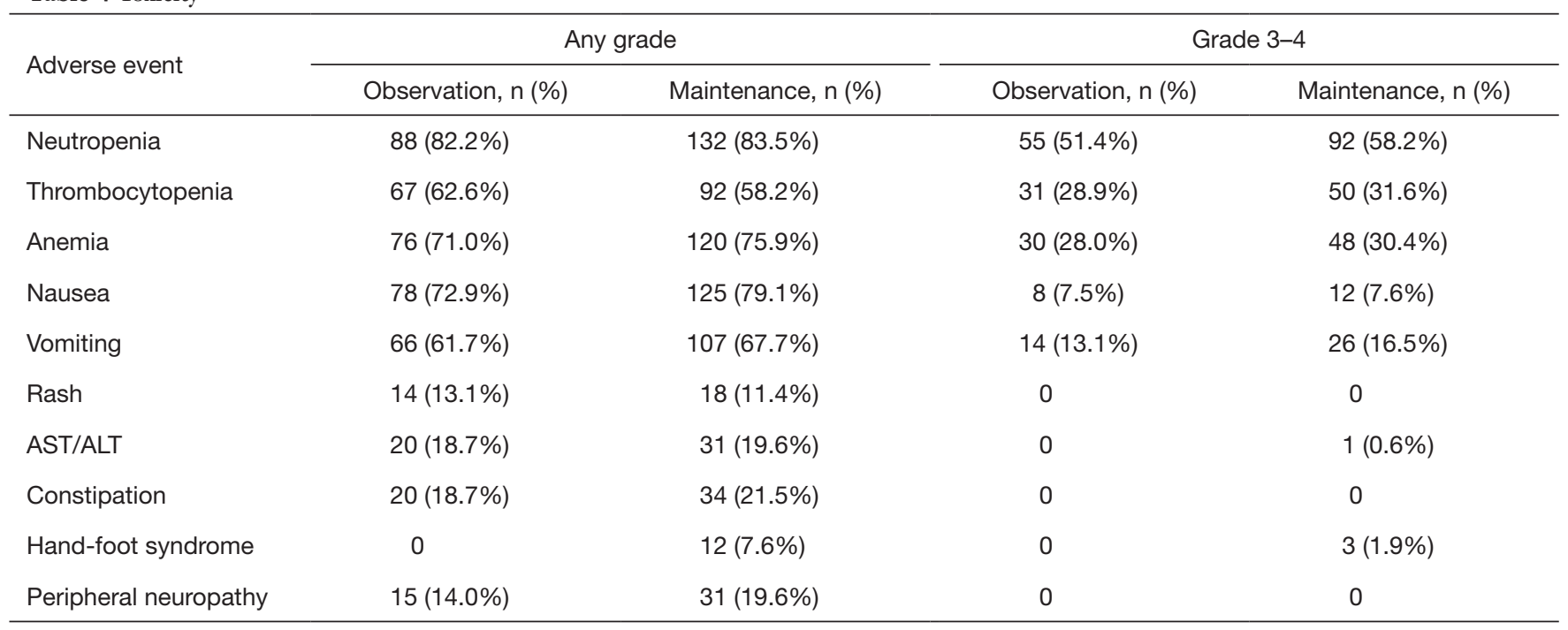

chemotherapy for mTNBC, it is still worth exploring how to further prolong the PFS and OS of patients based on the advantages of PBCT. In this study, we suggest that maintenance chemotherapy can prolong the PFS and OS of mTNBC patients who have achieved non-PD after first-line PBCT. Additionally, two- and single-drug 
maintenance chemotherapy strategies showed no difference in prolonging PFS. We also observed that there was no statistically significant difference in the incidence of adverse events between the two groups except hand-foot syndrome.

There is only one phase III clinical study, KCSGBR-0702, which has examined the use of maintenance treatment after first-line chemotherapy in the treatment of mTNBC (12). The maintenance treatment regimen in this trial was GP, which was identical to the first-line chemotherapy regimen. This study included not only patients with mTNBC but also HR-positive and HER2positive patients. This trial concluded that maintenance therapy resulted in better PFS and OS compared with observation, including that in the mTNBC subgroup. However, this study did not include the specific survival benefit data of the mTNBC subgroup, and the number of patients in this subgroup was small $(n=31)$. Although this trial could not provide direct evidence for the gemcitabinecisplatin regimen involved in our study, it confirmed the efficacy of doublet drug maintenance therapy in patients with mTNBC. Furthermore, a number of retrospective clinical studies have explored the value of different regimens of maintenance therapy for mTNBC. A retrospective clinical study established by the Peking University Cancer Institute in 2014 suggested that capecitabine maintenance therapy after initial capecitabine plus docetaxel chemotherapy in patients with mTNBC could significantly prolong the PFS with a favorable safety profile (10.1 vs. 6.7 months, $\mathrm{P}=0.032$ ) (14). Another retrospective study in 2016 by Ferrero et al. (13) found that patients with mTNBC who received first-line treatment with a three-drug regimen of paclitaxel, capecitabine, and bevacizumab followed by maintenance therapy with capecitabine and bevacizumab had a median PFS of 7.6 months (95\% CI: 6.3-9.0 months) and median OS of 19.2 months (95\% CI: 17.4-20.9 months).

Our study was the first to explore the relevance of maintenance therapy on patients' prognosis after first-line chemotherapy with PBCT in mTNBC. Compared with previous studies, we enrolled a larger number of patients and had a longer follow-up period. We also included various maintenance treatment regimens. Our study also had some limitations. Retrospective studies have poorer control over baseline characteristics and treatment standardization. To resolve this problem, we used a multivariate Cox proportional-hazards model to adjust for the treatment effects, eliminating the effects of other prognostic factors as much as possible, which led to relatively reliable conclusions. Our institution routinely used the original doublet-drug maintenance chemotherapy, and the number of patients in the single-drug maintenance group was relatively small. Due to the uneven distribution of the patients enrolled in each maintenance treatment regimen, we did not compare the specific maintenance treatment regimens. The results of this study only suggested that two- and single-drug maintenance chemotherapy strategies showed no difference in effect on prolonging PFS. This part will be further refined in our subsequent research.

Moreover, breast cancer is a highly heterogeneous disease (16), and the prognosis of breast cancers with different molecular subtypes is also quite different (17-19). There are several phase III clinical studies comparing different maintenance regimens in patients with HR-positive and HER2-positive advanced breast cancer (11,20-22). However, such clinical trials are still lacking for the population with mTNBC. In previous phase III clinical studies of first-line PBCT, the median PFS of the GP group in the CBCSG006 study was 7.73 months (8), and the median OS of the gemcitabine plus carboplatin group was 11.1 months (7). Compared with these studies, the survival benefits for our subjects have increased. Therefore, maintenance treatment for mTNBC will be a worthwhile research direction, and it may contribute to the precise treatment of mTNBC.

Besides chemotherapy, targeted therapy and immunotherapy are also two kinds of promising therapies for mTNBC (23). For example, PARP inhibitors and PD-1/PD-L1 inhibitors have achieved much progress in this field $(24,25)$. However, the hyperprogressors after immunotherapy, immature administration, and low response rate limit their roles in clinical application $(26,27)$. Thus, many clinical trials about PARP inhibitors and PD-1/PD-L1 inhibitors have been designed based on heavily pretreated mTNBC patients (28). Although the immunotherapy is the trend in tumor therapy, whether it could be the first-line therapy or even replace the chemotherapy is still unknown. At least chemotherapy will be the main treatment of $\mathrm{mTNBC}$ for a long period, and our study can provide a choice before new therapies are optimised.

Therefore, our results suggest that based on the advantages of PBCT, maintenance chemotherapy can significantly improve PFS and OS in patients with mTNBC after achieving disease control with first-line PBCT. There was no significant difference in the results on PFS of single and doublet-drug maintenance chemotherapies. This finding 
may play an important role in the management of mTNBC treatment and development of subsequent clinical trials.

\section{Acknowledgments}

Funding: This study was supported by the Shanghai Municipal Science and Technology Commission Guidance Project, P.R. China (contract No. 18411967800); Shanghai Natural Science Foundation (grant No. 17ZR1405700); research grant from Shanghai Hospital Development Center (grant No. SHDC12018X03); and CSCO-ROCHE Cancer Research Fund 2019 (grant No. Y-2019Roche-171).

\section{Footnote}

Reporting checklist: The authors have completed the STROBE reporting checklist. Available at http://dx.doi. org/10.21037/apm-20-578

Data Sharing Statement: Available at http://dx.doi. org/10.21037/apm-20-578

Conflicts of Interest: All authors have completed the ICMJE uniform disclosure form (available at http://dx.doi. org/10.21037/apm-20-578). JZ serves as an unpaid editorial board member of Annals of Palliative Medicine from January 2020 to January 2022. The other authors have no conflicts of interest to declare.

Ethical Statement: The authors are accountable for all aspects of the work in ensuring that questions related to the accuracy or integrity of any part of the work are appropriately investigated and resolved. The study was conducted in accordance with the Declaration of Helsinki (as revised in 2013). This retrospective study was approved by Fudan University Shanghai Cancer Center Medical Ethics Committee (No. 1412142-14) and informed consent was taken from all the patients.

Open Access Statement: This is an Open Access article distributed in accordance with the Creative Commons Attribution-NonCommercial-NoDerivs 4.0 International License (CC BY-NC-ND 4.0), which permits the noncommercial replication and distribution of the article with the strict proviso that no changes or edits are made and the original work is properly cited (including links to both the formal publication through the relevant DOI and the license). See: https://creativecommons.org/licenses/by-nc-nd/4.0/.

\section{References}

1. Foulkes WD, Smith IE, Reis JS. Triple-Negative Breast Cancer. N Engl J Med 2010;363:1938-48.

2. Kennecke H, Yerushalmi R, Woods R, et al. Metastatic Behavior of Breast Cancer Subtypes. J Clin Oncol 2010;28:3271-7.

3. Dent R, Trudeau M, Pritchard KI, et al. Triple-negative breast cancer: clinical features and patterns of recurrence. Clin Cancer Res 2007;13:4429-34.

4. Voduc KD, Cheang MCU, Tyldesley S, et al. Breast Cancer Subtypes and the Risk of Local and Regional Relapse. J Clin Oncol 2010;28:1684-91.

5. Kassam F, Enright K, Dent R, et al. Survival outcomes for patients with metastatic triple-negative breast cancer: implications for clinical practice and trial design. Clin Breast Cancer 2009;9:29-33.

6. Carey LA, Perou CM, Livasy CA, et al. Race, breast cancer subtypes, and survival in the Carolina Breast Cancer Study. JAMA 2006;295:2492-502.

7. O'Shaughnessy J, Schwartzberg L, Danso MA, et al. Phase III Study of Iniparib Plus Gemcitabine and Carboplatin Versus Gemcitabine and Carboplatin in Patients With Metastatic Triple-Negative Breast Cancer. J Clin Oncol 2014;32:3840-U232.

8. $\mathrm{Hu} \mathrm{XC}, \mathrm{Zhang} \mathrm{J}, \mathrm{Xu} \mathrm{BH}$, et al. Cisplatin plus gemcitabine versus paclitaxel plus gemcitabine as first-line therapy for metastatic triple-negative breast cancer (CBCSG006): a randomised, open-label, multicentre, phase 3 trial. Lancet Oncol 2015;16:436-46.

9. Sánchez-Muñoz A, Pérez-Ruiz E, Ribelles N, et al. Maintenance treatment in metastatic breast cancer. Expert Rev Anticancer Ther 2008;8:1907-12.

10. Rossi S, Schinzari G, Basso M, et al. Maintenance hormonal and chemotherapy treatment in metastatic breast cancer: a systematic review. Future Oncol 2016;12:1299-307.

11. Dufresne A, Pivot X, Tournigand C, et al. Maintenance hormonal treatment improves progression free survival after a first line chemotherapy in patients with metastatic breast cancer. Int J Med Sci 2008;5:100-5.

12. Park YH, Jung KH, Im SA, et al. Phase III, multicenter, randomized trial of maintenance chemotherapy versus observation in patients with metastatic breast cancer after achieving disease control with six cycles of gemcitabine plus paclitaxel as first-line chemotherapy: KCSGBR07-02. J Clin Oncol 2013;31:1732-9.

13. Ferrero JM, Hardy-Bessard AC, Capitain O, et al. Weekly paclitaxel, capecitabine, and bevacizumab with 
maintenance capecitabine and bevacizumab as first-line therapy for triple-negative, metastatic, or locally advanced breast cancer: Results from the GINECO A-TaXel phase 2 study. Cancer 2016;122:3119-26.

14. Liang X, Di L, Song G, et al. Capecitabine maintenance therapy for XT chemotherapy-sensitive patients with metastatic triple-negative breast cancer. Chin J Cancer Res 2014;26:550-7.

15. Paluch-Shimon S, Pagani O, Partridge AH, et al. ESOESMO 3rd international consensus guidelines for breast cancer in young women (BCY3). Breast 2017;35:203-17.

16. Kennecke H, Yerushalmi R, Woods R, et al. Metastatic behavior of breast cancer subtypes. J Clin Oncol 2010;28:3271-7.

17. Alanko A, Heinonen E, Scheinin T, et al. Significance of estrogen and progesterone receptors, disease-free interval, and site of first metastasis on survival of breast cancer patients. Cancer 1985;56:1696-700.

18. Chia S, Norris B, Speers C, et al. Human epidermal growth factor receptor 2 overexpression as a prognostic factor in a large tissue microarray series of node-negative breast cancers. J Clin Oncol 2008;26:5697-704.

19. Chiang AC, Massague J. Molecular basis of metastasis. N Engl J Med 2008;359:2814-23.

20. Slamon DJ, Leyland-Jones B, Shak S, et al. Use of chemotherapy plus a monoclonal antibody against HER2 for metastatic breast cancer that overexpresses HER2. N Engl J Med 2001;344:783-92.

21. Kaufman B, Mackey JR, Clemens MR, et al. Trastuzumab Plus Anastrozole Versus Anastrozole Alone for the

Cite this article as: Chen $\mathrm{Y}$, Zhang J, Hu XC, Wang BY, Wang ZH, Wang LP, Cao J, Tao ZH, Du YQ, Zhao YN, Gong CC, Jin J. Maintenance chemotherapy is effective in patients with metastatic triple negative breast cancer after first-line platinum-based chemotherapy. Ann Palliat Med 2020;9(5):3018-3027. doi: 10.21037/apm-20-578
Treatment of Postmenopausal Women With Human Epidermal Growth Factor Receptor 2-Positive, Hormone Receptor-Positive Metastatic Breast Cancer: Results From the Randomized Phase III TAnDEM Study. J Clin Oncol 2009;27:5529-37.

22. Swain SM, Baselga J, Kim SB, et al. Pertuzumab, trastuzumab, and docetaxel in HER2-positive metastatic breast cancer. N Engl J Med 2015;372:724-34.

23. Bianchini G, Balko JM, Mayer IA, et al. Triplenegative breast cancer: challenges and opportunities of a heterogeneous disease. Nat Rev Clin Oncol 2016;13:674-90.

24. Robson M, Im SA, Senkus E, et al. Olaparib for Metastatic Breast Cancer in Patients with a Germline BRCA Mutation. N Engl J Med 2017;377:523-33.

25. Nanda R, Chow LQ, Dees EC, et al. Pembrolizumab in Patients With Advanced Triple-Negative Breast Cancer: Phase Ib KEYNOTE-012 Study. J Clin Oncol 2016;34:2460-7.

26. Kato S, Goodman A, Walavalkar V, et al. Hyperprogressors after Immunotherapy: Analysis of Genomic Alterations Associated with Accelerated Growth Rate. Clin Cancer Res 2017;23:4242-50.

27. Saada-Bouzid E, Defaucheux C, Karabajakian A, et al. Hyperprogression during anti-PD-1/PD-L1 therapy in patients with recurrent and/or metastatic head and neck squamous cell carcinoma. Ann Oncol 2017;28:1605-11.

28. Emens LA. Breast Cancer Immunotherapy: Facts and Hopes. Clin Cancer Res 2018;24:511-20. 